\title{
Evaluation of the SD Bioline TB Ag MPT64 test for identification of Mycobacterium tuberculosis complex from liquid cultures in Southwestern Uganda
}

\begin{tabular}{|c|c|}
\hline \multicolumn{2}{|c|}{$\begin{array}{l}\text { Authors: } \\
\text { Patrick Orikiriza }^{1,2} \\
\text { Dan Nyehangane }^{1} \\
\text { Daniel Atwine }{ }^{1} \\
\text { John J. Kisakye }^{3} \\
\text { Kennedy Kassaza }^{1} \\
\text { Juliet-Mwanga Amumpaire } \\
\text { Yap Boum } \text { II }^{1}\end{array}$} \\
\hline $\begin{array}{l}\text { Affiliations: } \\
{ }^{1} \text { Epicentre Ml } \\
\text { Centre, Mbar }\end{array}$ & $\begin{array}{l}\text { arara Research } \\
\text { ra, Uganda }\end{array}$ \\
\hline \multicolumn{2}{|c|}{$\begin{array}{l}{ }^{2} \text { Department of Microbiology, } \\
\text { Faculty of Medicine, Mbarara } \\
\text { University of Science and } \\
\text { Technology, Mbarara, Uganda }\end{array}$} \\
\hline \multicolumn{2}{|c|}{$\begin{array}{l}{ }^{3} \text { Department of Biological } \\
\text { Sciences, College of Natural } \\
\text { Sciences, Makerere } \\
\text { University, Kampala, Uganda }\end{array}$} \\
\hline \multicolumn{2}{|c|}{$\begin{array}{l}\text { Corresponding author: } \\
\text { Patrick Orikiriza, } \\
\text { patrick.orikiriza@epicentre. } \\
\text { msf.org }\end{array}$} \\
\hline \multicolumn{2}{|c|}{$\begin{array}{l}\text { Dates: } \\
\text { Received: } 20 \text { Oct. } 2015 \\
\text { Accepted: } 23 \text { May } 2016 \\
\text { Published: } 31 \text { Mar. } 2017\end{array}$} \\
\hline \multicolumn{2}{|c|}{$\begin{array}{l}\text { How to cite this article: } \\
\text { Orikiriza P, Nyehangane D, } \\
\text { Atwine D, et al. Evaluation of } \\
\text { the SD Bioline TB Ag MPT64 } \\
\text { test for identification of } \\
\text { Mycobacterium tuberculosis } \\
\text { complex from liquid cultures } \\
\text { in Southwestern Uganda. } \\
\text { Afr J Lab Med. 2017;6(2), } \\
\text { a383. https://doi. } \\
\text { org/10.4102/ajlm.v6i2.383 }\end{array}$} \\
\hline $\begin{array}{l}\text { Copyright: } \\
\text { (C) 2017. The } \\
\text { Licensee: AOS } \\
\text { is licensed un } \\
\text { Creative Com } \\
\text { Attribution Lic }\end{array}$ & $\begin{array}{l}\text { uthors. } \\
\text { S. This work } \\
\text { er the } \\
\text { lons } \\
\text { ense. }\end{array}$ \\
\hline Read online: & \\
\hline 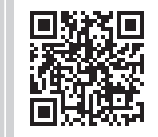 & $\begin{array}{l}\text { Scan this QR } \\
\text { code with your } \\
\text { smart phone or } \\
\text { mobile device } \\
\text { to read online. }\end{array}$ \\
\hline
\end{tabular}

Background: To confirm presence of Mycobacterium tuberculosis complex, some tuberculosis culture laboratories still rely on para-nitrobenzoic acid (PNB), a traditional technique that requires sub-culturing of clinical isolates and two to three weeks to give results. Rapid identification tests have improved turnaround times for mycobacterial culture results. Considering the challenges of the PNB method, we assessed the performance of the SD Bioline TB Ag MPT64 assay by using PNB as gold standard to detect M. tuberculosis complex from acid-fast bacilli (AFB) positive cultures.

Objectives: The aim of this study was to determine the sensitivity, specificity and turnaround time of the SD MPT64 assay for identification of M. tuberculosis complex, in a setting with high prevalence of tuberculosis and HIV.

Methods: A convenience sample of 690 patients, with tuberculosis symptoms, was enrolled at Epicentre Mbarara Research Centre between April 2010 and June 2011. The samples were decontaminated using NALC-NaOH and re-suspended sediments inoculated in Mycobacterium Growth Indicator Tubes (MGIT) media, then incubated at $37^{\circ} \mathrm{C}$ for a maximum of eight weeks. A random sample of 50 known negative cultures and 50 non-tuberculous mycobacteria isolates were tested for specificity, while sensitivity was based on AFB positivity. The time required from positive culture to reporting of results was also assessed with PNB used as the gold standard.

Results: Of the 138 cultures that were AFB-positive, the sensitivity of the SD MPT64 assay was 100.0\% [95\% CI: 97.3 - 100] and specificity was 100.0\% (95\% CI, $96.4-100)$. The median time from a specimen receipt to confirmation of strain was 10 days [IQR: 8-12] with SD MPT64 and 24 days [IQR: 22-26] with PNB.

Conclusion: The SD MPT64 assay is comparable to PNB for identification of M. tuberculosis complex and reduces the time to detection.

\section{Introduction}

Proper diagnosis is the first step toward better management and prevention of tuberculosis transmission. The Stop TB Partnership has recently described the actions and resources needed to end tuberculosis in the world by $2030^{1}$. The world is now focusing on a 'paradigm shift' that will see countries improve case finding and decrease tuberculosis incidence rates by at least $10 \%$ annually. In order to achieve this global plan to end tuberculosis, all healthcare practitioners will be required to ensure that $90 \%$ of vulnerable groups are screened, $90 \%$ of those diagnosed are started on treatment and $90 \%$ are successfully treated. As such, one of the global priorities is on diagnosis. ${ }^{1}$ Discovery, development and rapid uptake of new tools and interventions have been highlighted as major requirements for the success of this plan.

Currently, significant success has been noted with the integration of the Xpert ${ }^{\circledR}$ MTB/RIF (Cepheid, United States) assay into clinical practice. ${ }^{2}$ While this tool has the advantage of detecting Mycobacterium tuberculosis complex and mutations associated with rifampicin resistance, within approximately two hours ${ }^{2}$, challenges exist in low-resource settings related to instrument breakdown, inconsistent electric power supply, delayed maintenance, irregular supply of cartridges, limited machine capacity (four tests every two hours) and errors. ${ }^{2}$ Despite the new tool, approximately three million tuberculosis cases annually are not diagnosed, ${ }^{1}$ thus causing a public health challenge.

Tuberculosis and HIV form a deadly synergy, ${ }^{3}$ yet the number of bacilli in the sputum samples of co-infected patients is usually low. As a result, countries including Uganda have adopted national 
tuberculosis programme guidelines that prioritise the use of the Xpert $^{\circledR}$ MTB/RIF assay on sputum from vulnerable groups such as children and on acid-fast bacilli (AFB)-smearnegative, HIV-positive patients with signs and symptoms of tuberculosis. ${ }^{4}$ However, the majority of persons with presumptive tuberculosis do not necessarily know their HIV status and may not be eligible for the Xpert ${ }^{\circledR}$ MTB/RIF assay.

Bacteriological confirmation of tuberculosis by culture isolation currently remains the diagnostic reference standard recommended by the World Health Organization. ${ }^{5}$ However, even with a positive liquid culture there is need to differentiate $M$. tuberculosis complex and non-tuberculous mycobacteria (NTM). Although this can easily be achieved by the current molecular methods such as line probe assays, these are complex and bear high infrastructural and human resource requirements. ${ }^{6}$

Traditional methods, such as the use of para-nitrobenzoic acid (PNB) on Ziehl-Neelsen (ZN) positive cultures, are simple, but require pure isolates, which delays results (2-3 weeks). ${ }^{6,7}$ This delay impacts clinical management of the patient and potentially prolongs transmission among contacts of tuberculosis patients. There are additional costs associated with incubation requirements, such as staff time, space in the incubator, electricity and other factors that are not always considered. $^{7}$

Recently, rapid methods of identifying $M$. tuberculosis complex from AFB-positive cultures have been developed. These rely on chromatographic detection of MPT64, a protein that is produced by $M$. tuberculosis complex during its metabolism in cultures. ${ }^{8}$ Among these, the two commonly-available methods include the Capilia TB-Neo assay (Tauns Laboratories, Inc., Numazu, Japan) and the SD Bioline TB Ag MPT64 assay (Standard Diagnostics, Yongin-si, Gyeonggi-do, Republic of Korea; hereafter, SD MPT64 [assay]). These assays have the advantage of being inexpensive, easy to use and readily available, even in low-resource settings. ${ }^{9}$ They are easily stored at room temperature and allow for results from positive cultures within 15 minutes. ${ }^{10}$ Available data from evaluation of the Capilia TB-Neo assay indicate that it has excellent sensitivity and specificity. ${ }^{911,12}$ Few studies have been done on the SD MPT64 assay in field settings. ${ }^{13,14,15}$ Nevertheless, from the results of these studies, there is general agreement that these methods are suitable replacements for the traditional methods.

The aim of this study was to determine the sensitivity, specificity and turnaround time of the SD MPT64 assay for identification of $M$. tuberculosis complex, in a setting with high prevalence of tuberculosis and HIV.

\section{Methods}

\section{Ethical considerations}

The samples were obtained from patients enrolled at the Epicentre Mbarara Research Centre with approval from the Faculty Research and Ethics committee and the Institutional Review Board at Mbarara University of Science and
Technology, as well as the Uganda National Council for Sciences and Technology. All patients signed an informed consent form to participate in the main study and allow further testing on the samples and isolates.

\section{Study design}

Samples for this cross-sectional study were obtained from patients with signs and symptoms of pulmonary tuberculosis according to World Health Organization guidelines. ${ }^{16}$ We enrolled 690 patients at the Epicentre Mbarara Research Centre, Mbarara, Uganda, between April 2010 and June 2011 in a separate study to assess the utility of colorimetric methods to detect $M$. tuberculosis complex in patients with suspected tuberculosis. ${ }^{17}$ Patients were eligible if they reported a cough for more than two weeks, were at least 15 years of age and signed an informed consent. After consenting, patients' samples were collected, stored in a cool box and then transported immediately to the laboratory. They were decontaminated using the $\mathrm{N}$-acetyl L-cysteine (NALC)-NaOH procedure. ${ }^{18}$ The concentrated sediments were homogenised with phosphate buffer, inoculated in Mycobacterium Growth Indicator Tubes (MGIT) (BD, Franklin Lakes, New Jersey, United States) medium and then incubated at $37^{\circ} \mathrm{C}$ for up to eight weeks. MGIT cultures were read daily on a manual reader until growth was detected. To check for the presence of AFB all positive cultures were tested by Ziehl-Neelsen (ZN) smear microscopy; to check for contamination, positive cultures were tested by blood agar. The Epicentre Laboratory participates in an external quality assurance scheme for culture with the National Health Laboratory Services, South Africa.

For this study, a convenience sample of 138 AFB-positive cultures was considered for testing with SD MPT64 and PNB. The two tests were performed immediately on the isolate according to the manufacturer's recommendations and laboratory protocol. If $\mathrm{AFB}$ was present together with contamination, the isolate was re-decontaminated with $\mathrm{NaOH}$, sub-cultured in a fresh culture tube and monitored for pure growth before repeating PNB.

To determine specificity, we randomly selected 50 knownnegative MGIT samples from the study, which were tested independently with SD MPT64 and PNB. To confirm ability of the test to rule out NTMs, an additional sample of 50 known isolates of Mycobacterium fortuitum were subcultured into MGIT using a standard inoculum of McFarland 0.5. These were monitored daily until positivity was detected by the MGIT instrument. We performed the SD MPT64 assay and PNB on each of them as described above for the MGITnegative cultures (Figure 1).

The PNB media was prepared locally, stored between $2{ }^{\circ} \mathrm{C}-8{ }^{\circ} \mathrm{C}$ and quality controlled according to our standard operating procedures. The test required two Lowenstein Jensen slants one with and another without PNB reagent, the latter serving as negative control. Both slants were inoculated with $500 \mu \mathrm{L}$ of standard inoculum, incubated at $37^{\circ} \mathrm{C}$ and read weekly until colonial growth was observed. Identification 


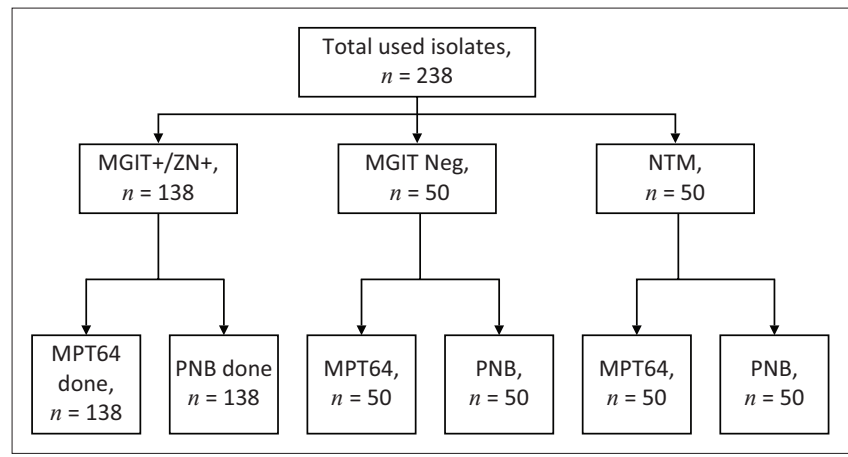

PNB, para-nitrobenzoic acid; NTM, non-tuberculous mycobacteria; ZN, Ziehl-Neelsen. PNB was used as the gold standard. Included in the study were $138 \mathrm{ZN}$-positive cultures, 50 NTM and 50 negative cultures.

FIGURE 1: Study Profile.

of $M$. tuberculosis complex was made based on presence of growth in the control tube (without PNB), with no growth in the tube with PNB.

Manufacturer instructions were followed in performing the SD MPT64 assay. In brief, $100 \mu \mathrm{L}$ of mixed MGIT culture was added directly to the test cartridge and allowed to flow chromatographically for 15 minutes. A positive result was indicated by a red band on the test window in addition to the control band. Data were collected using case report forms and double entered using Voozanoo software version 2 (EpiConcept, Paris, France). Turnaround time was calculated by measuring the time taken from specimen receipt to reporting of a positive SD MPT64 or PNB assay. Ease of use of the techniques was determined through a questionnaire that was filled out by all technicians performing the tests.

\section{Statistical analyses}

Statistical analysis was performed using Stata SE v.11 software (College Station, Texas, United States, 2009). We considered a sample positive with $M$. tuberculosis complex when confirmed by the PNB gold standard and negative if not confirmed positive for M. tuberculosis complex by the gold standard. For SD MPT64, performance was calculated by estimating the sensitivity, specificity and 95\% confidence interval.

\section{Results}

The median age (years) and interquartile range (IQR) was 38 (30-48) with a gender ratio (male/female) of 49/51 and HIV positivity of $58.6 \%$. Of the 138 AFB-positive MGIT cultures among the 690 patients included in the study, 136 cultures (98.6\%) were confirmed positive for $M$. tuberculosis complex by both SD MPT64 and PNB, and two as NTM (Table 1). This gave a sensitivity of $100 \%$ [95\% CI: 97.3-100]. All the known negative cultures and NTM were reported as negative for $M$. tuberculosis complex, giving a specificity of $100.0 \%$ [95\% CI: 96.4-100], respectively.

The median time from specimen receipt to confirmed identification of $M$. tuberculosis complex was 10 days [IQR: 8-12 days] with SD MPT64 and 24 days [IQR: 22-26] with PNB. All the technicians who performed the laboratory
TABLE 1: Cross tabulation of SD MPT64 assay and para-nitrobenzoic acid.

\begin{tabular}{lcccc}
\hline SD MPT64 assay & \multicolumn{4}{c}{ Reference standard (PNB) } \\
\cline { 2 - 5 } & Negative & M. tuberculosis & NTM & Total \\
\hline Positives & 0 & 0 & 0 & 0 \\
Negative & 0 & 136 & 0 & 136 \\
M. tuberculosis & 0 & 0 & 2 & 2 \\
NTM & & & & \\
Negatives & 50 & 0 & 0 & 50 \\
Negative & 0 & 0 & 0 & 0 \\
M. tuberculosis & 0 & 0 & 0 & 0 \\
NTM & & & & \\
NTM & 0 & 0 & 0 & 0 \\
Negative & 0 & 0 & 0 & 0 \\
M. tuberculosis & 0 & 0 & 50 & 50 \\
NTM & & 0 &
\end{tabular}

PNB, para-nitrobenzoic acid; NTM, non-tuberculous mycobacteria; ZN, Ziehl-Neelsen.

tests reported that the SD MPT64 assay was easy to use and did not require additional training other than the standard operating procedure. This was not the case with the PNB method.

\section{Discussion}

Few studies have evaluated the performance of the SD MPT64 assay in field settings with high tuberculosis and HIV burdens. This study confirms prior evaluations and increases the evidence that the test has excellent sensitivity and specificity in identifying $M$. tuberculosis complex using PNB as a gold standard, in a Ugandan field setting where there is high tuberculosis and HIV co-infection. There have been previous evaluations in various countries using different gold standards. All the investigations have shown that the technique has high sensitivity and specificity. One study used reference bacterial strains and Mycobacterium bovis field isolates from animals and found a sensitivity of $96.5 \%$ [95\% CI: 91.2-99.0] and specificity of 100\% [95\% CI: 96.7-100]. ${ }^{19}$ The same group also found a high positive predictive value of 100\% [95\% CI: 96.7-100], and a negative predictive value of 92.9\% [95\% CI: 82.7-98.0].

The SD MPT64 assay was evaluated on a large number of clinical isolates in India and performed with 100.0\% sensitivity and specificity. ${ }^{9}$ Another recent study in India used isolates from extra-pulmonary and NTM samples and found SD MPT64 to have a 100.0\% sensitivity and specificity compared with conventional tests such as niacin, nitrate reduction and PNB. ${ }^{13}$ Although the sample size was small, they demonstrated the accuracy, cost effectiveness and early identification of $M$. tuberculosis complex in patients with paucibacillary extra-pulmonary tuberculosis.

The performance of the Capilia TB-Neo assay has been evaluated extensively. One study evaluated its performance using reference strains of $M$. tuberculosis complex, NTM and other non-related bacteria. They used nucleic acid assays in addition to the Capilia TB-Neo and SD MPT64 assays. The Capilia TB-Neo assay had $99.6 \%$ sensitivity and 100\% specificity, unlike the SD MPT64 assay which had several false positive results with the NTM that were attributed to a high 
concentration of the bacterial antigen ${ }^{20}$. This poor specificity, however, was not observed in our study possibly due to only one NTM species evaluated. In Uganda, a study done in Kampala city provided evidence that the Capilia TB-Neo assay can be used to identify M. tuberculosis complex successfully from blood cultures. ${ }^{12}$ They found an overall sensitivity of $98.4 \%$ and overall specificity of $97.6 \%$ compared to polymerase chain reaction, which supports its use for blood culture isolates.

\section{Limitations}

This study was nested within a colorimetric study and thus relied on study design, population selection and sample size of the primary study. The lack of other NTM strains limited exploration of false positivity as reported in other studies.

\section{Recommendations}

Basing on our findings, we recommend that the SD Bioline TB Ag MPT64 test be used in place of PNB for identification of M. tuberculosis complex from liquid cultures.

\section{Conclusion}

The SD Bioline MPT64 assay has good sensitivity and specificity for rapid discrimination between M. tuberculosis complex and NTM in clinical isolates. It also has a short turnaround time in confirming $M$. tuberculosis complex in AFB-positive cultures. In addition, the technicians reported that the test is easy to perform.

\section{Acknowledgements}

We would like to express our gratitude and thanks to the study participants. We also thank the Epicentre nurses and laboratory personnel, especially John Mary Ngattu, Henry Munyambabazi and Esther Turyashemererwa, who participated in the data collection. We also acknowledge Médecins Sans Frontières for funding the study that generated this data. Special thanks to Christopher C. Moore for a thorough review of this manuscript. Finally, we are grateful to the Uganda Research Student Support Fund for linking the study team.

\section{Competing interests}

The authors declare that they have no financial or personal relationships which may have inappropriately influenced them in writing this article.

\section{Sources of support}

This work was made possible with funding from Médecins Sans Frontières. Additional support was received from the Uganda Research Student Support Fund, an initiative that was conceived to fund global students' research and to link programmes between Ugandan universities and several international partners to conduct research on global health issues. The funders had no role in study design, data collection and analysis, decision to publish, or preparation of the manuscript.

\section{Authors' contributions}

P.O., D.N., J.J.K. and Y.B. conceived the idea and designed the experiments. P.O., D.N., J.J.K., K.K. and Y.B. performed the laboratory experiments. P.O., D.N., J.M.A. and D.A. analysed the data. P.O., D.N., J.J.K., K.K., J.-M.A. and Y.B. wrote and reviewed the manuscript. All authors approved the final version of the manuscript. P.O. was the project leader, while Y.B. and J.M.A. were project supervisors. All authors made conceptual contributions and were responsible for experimental and project design. P.O., D.N. and K.K. performed some of the experiments. P.O., D.N. and K.K. prepared the samples and calculations were performed by D.A.

\section{References}

1. Stop TB. Partnership: A partnership hosted by United Nations at UNOPS; 2015. http://stoptb.org/assets/documents/global/plan/GlobalPlanToEndTB TheParadigmShift_2016-2020_StopTBPartnership.pdf

2. Boehme CC, Nicol MP, Nabeta P, et al. Feasibility, diagnostic accuracy, and effectiveness of decentralised use of the Xpert MTB/RIF test for diagnosis of tuberculosis and multidrug resistance: A multicentre implementation study. Lancet. 2011;377:1495-1505. http://dx.doi.org/10.1016/S0140-6736(11)60438-8

3. Kwan CK and Ernst JD. HIV and tuberculosis: a deadly human syndemic. Clin Microbiol Rev. 2011 Apr;24(2):351-76. doi:10.1128/CMR.00042-10

4. Mugabe Frank: Program Manager Uganda National Tuberculosis and Leprosy Program, Ministry of Health. Uganda Making Xpert MTB/RIF change the game for PLHIV. Presentation. GLI -Meeting Geneva 30th April -2nd May 2014.

5. Global Tuberculosis Report. 20th ed. Geneva, Switzerland: WHO/HTM/ TB/2015.22; 2015

6. World Health Organization. Molecular line probe assays for rapid screening of patients at risk of multidrug-resistant tuberculosis (MDR-TB). Policy statement. Geneva, Switzerland: WHO; 2008.

7. World Health Organization. Laboratory services in Tuberculosis control. Part III culture. Geneva, Switzerland: WHO; 1998. WHO/TB/98.258. Original: English.

8. Hasegawa $N$, Miura T, Ishii $K$, et al. New simple and rapid test for culture confirmation of Mycobacterium tuberculosis complex: A multicenter study. J Clin Microbiol. 2002;40:908-912. http://dx.doi.org/10.1128/JCM.40.3.908-912.2002

9. Shenoy VP, Mukhopadhyay C. Rapid Immunochromatographic Test for the Identification and Discrimination of Mycobacterium tuberculosis Complex Isolates from Non-tuberculous Mycobacteria. J Clin Diagn Res. 2014;8(4):DC13-DC15. http://dx.doi.org/10.7860/JCDR/2014/7098.4253

10. Gaillard T, Fabre M, Martinaud C, Vong R, Brisou P, Soler C. Assessment of the SD Bioline Ag MPT64 Rapid TM and the MGIT TM TBc identification tests for the diagnosis of tuberculosis. Diagn Microbiol Infect Dis. 2011;70:154-156. http:// dx.doi.org/10.1016/j.diagmicrobio.2010.12.011

11. Maurya AK, Nag VL, Kant S, et al. Evaluation of an immunochromatographic test for discrimination between Mycobacterium tuberculosis complex and non tuberculous mycobacteria in clinical isolates from extra-pulmonary tuberculosis. Indian J Med Res. 2012;135:901-906.

12. Muchwa C, Akol J, Etwom A, et al. Evaluation of Capilia TB assay for rapid identification of Mycobacterium tuberculosis complex in BACTEC MGIT 960 and BACTEC 9120 blood cultures. BMC Res Notes. 2012;5:44. http://dx.doi. org/10.1186/1756-0500-5-44

13. Kandhakumari G, Stephen S. Extra pulmonary tuberculosis: Rapid identification of Mycobacterium tuberculosis grown in Mycobacterium growth indicator tube 960 and Lowenstein-Jensen media, employing Standard diagnostics Bioline Microbiol. 2015;33(5):122-125. http://dx.doi.org/10.4103/0255-0857.150912

14. Kumar VG, Urs TA, Ranganath RR. MPT 64 Antigen detection for rapid confirmation of M. tuberculosis isolates. BMC Res Notes. 2011;24:79. http://dx.doi. org/10.1186/1756-0500-4-79

15. Kanade S, Nataraj G, Suryawanshi R, Mehta P. Utility of MPT 64 antigen detection assay for rapid characterization of mycobacteria in a resource constrained setting. Indian J Tuberc. 2012;59:92-96.

16. World Health Organization. Treatment of tuberculosis: Guidelines. 4th ed. Geneva, Switzerland: WHO; 2010.

17. Boum $Y$, Orikiriza P, Rojas-Ponce $G$, et al. Use of colorimetric culture methods for detection of Mycobacterium tuberculosis complex isolates from sputum samples in resource-limited settings. J Clin Microbiol. 2013;51:2273-2279. http://dx.doi. org/10.1128/JCM.00749-13

18. Kent PT, Kubica GP. 1985. Public health mycobacteriology: A guide for the level III laboratory. U.S. Department of Health and Human services, Centers for Disease Control and Prevention, Atlanta, GA.

19. Byeon HS, Ji MJ, Kang SS, et al. Performance of the SD Bioline TB Ag MPT64 Rapid Test for rapid confirmation of Mycobacterium bovis isolates from animals. J Vet Sci. 2014;16:31-35. http://dx.doi.org/10.4142/jvs.2015.16.1.31

20. Chikamatsu K, Aono A, Yamada H, et al. Comparative evaluation of three immunochromatographic identification tests for culture confirmation of Mycobacterium tuberculosis complex. BMC Infect Dis. 2014;1:54 